\title{
Correction to: Seasonal variations of PCDD/Fs in fishes: inferring a hidden exposure route from Na-PCP application for schistosomiasis control
}

\author{
Yuming Hu • Chuanzhu Yi • Jingguang Li • Xiaohong Shang • Zimin Li • \\ Xiaochen Yin • Bo Chen • Yuechan Zhou • Yinyin Zhang • Yongning Wu $(\mathbb{D}$
}

Published online: 7 May 2018

(C) Springer International Publishing AG, part of Springer Nature 2018

\section{Correction to: Environ Monit Assess (April 2018) 190, Issue 4:232 \\ https://doi.org/10.1007/s10661-018- 6533-6}

The original version of this article unfortunately contained an error in the affiliation section.

The affiliation of the first, second, fifth, sixth, eighth and ninth author should have been Hunan Provincial Center for Disease and Prevention, Changsha, 410005, China instead of China National Center for Food Safety Risk Assessment, Beijing, China.

The online version of the original article can be found at https://doi.org/10.1007/s10661-018-6533-6

Y. Hu · C. Yi · Z. Li • X. Yin · Y. Zhou · Y. Zhang

Hunan Provincial Center for Disease and Prevention, Changsha 410005, China

J. Li $\cdot$ X. Shang $\cdot$ Y. Wu $(\bowtie)$

China National Center for Food Safety Risk Assessment,

Beijing, China

e-mail: wuyongning@cfsa.net.cn

B. Chen

Department of Chemistry of School of Chemistry and Chemical Engineering of Hunan Normal University, Changsha, China
The affiliation of the seventh author should have been Department of Chemistry of School of Chemistry and Chemical Engineering of Hunan Normal University, Changsha, China instead of China National Center for Food Safety Risk Assessment, Beijing, China.

The corrected affiliations are shown below. 